\title{
The Incentive Mechanism of Government to Bank in Loan Risk Compensation Mechanism for Technological SMEs
}

\author{
Mu Zhang ${ }^{1, *}$ Zhi-yuan L ̈̈̈ ${ }^{1,2}$ \\ ${ }^{1}$ School of Big Data Application and Economics, Guizhou University of Finance and Economics \\ ${ }^{2}$ Guizhou Institution for Technology Innovation \& Entrepreneurship Investment, Guizhou University of Finance and \\ Economics \\ *Corresponding author. Email: rim_007@163.com
}

\begin{abstract}
Establishing and perfecting the incentive mechanism of government to bank in loan risk compensation mechanism for technological SMEs is of great significance to improving the use efficiency of loan risk compensation funds for technological SMEs. Based on loans origination operation and bad debts write-off management operation, a multi-task principal-agent model for bank in loan risk compensation mechanism for technological SMEs was constructed, so that the optimal incentive conditions of bank were solved in two aspects: the incentive cost functions are inter-independent or inter-dependent. The results show, both the rational government and bank are induced by reality to complete the loans origination operation using more energy and time, but the bad debts write-off management operation is ignored to some extent. Therefore, the government should establish the quality evaluation system of bad debts write-off management for bank, as a result, the effort degree of bank in process of completing the bad debts write-off management operation can be directly observed. On the basis, paying equal attention to the performance of loans origination operation and bad debts write-off management operation, the incentive mechanism of government to bank will be constructed.
\end{abstract}

Keywords: technological SMEs, loan risk compensation mechanism, government, bank, multi-task principal-agent model, optimal incentive contract

\section{科技型中小企业贷款风险补偿机制中政府对银行的激 励问题研究}

张 目 $^{1, *}$ 吕知远 ${ }^{1,2}$

\author{
${ }^{1}$ 大数据应用与经济学院, 贵州财经大学 \\ 2 贵州科技创新创业投资研究院, 贵州财经大学 \\ *通讯作者。电子邮箱： rim_007@163.com
}

\section{摘要}

建立健全科技型中小企业贷款风险补偿机制中政府对银行的激励机制, 对提高科技型中小企业贷款风险补偿 资金使用效率具有重要意义。基于贷款发放和呆账核销管理两项工作任务, 构建银行在科技型中小企业贷款 风险补偿机制中的多任务委托代理模型, 求解出激励成本函数相互独立和相互依存两种情形下的银行最优化 激励条件。结果显示，理性的政府和银行，都会被现实所诱导，用更多的精力和时间去完成贷款发放任务， 而花在呆账核销管理任务上的努力则相对较少。因此，政府有必要建立银行呆账核销管理质量评估体系，对 银行在完成呆账核销管理任务过程中的努力程度进行直接观测。在此基础上，政府可以建立贷款发放业绩和 呆账核销管理业绩并重的银行激励机制。 
关键字: 科技型中小企业，贷款风险补偿机制，政府，银行，多任务委托代理模型，最优激励合约。

\section{1. 引言}

科技型中小企业贷款风险补偿资金，是鼓励和 促进银行增加科技型中小企业贷款的政府引导性专 项扶持资金，主要用于对银行新增科技型中小企业 贷款产生的风险进行补偿, 或者对银行产生的科技 型中小企业贷款呆账给予补贴, 也可以对科技型中 小企业贷款增加较多、风险控制较好的银行给予适 当的奖励。实际上, 在 “市场运作、风险共担” 的 科技型中小企业贷款风险补偿机制中, 政府与银行 之间构成了委托代理关系, 因此, 建立健全政府激 励银行努力、高效地运作科技型中小企业信贷业务 的机制，对提高科技型中小企业贷款风险补偿资金 使用效率、促进科技和金融结合、推进自主创新具 有重要意义。

目前, 国内外相关研究主要集中于对科技型中 小企业贷款风险补偿政策及其效果、贷款风险补偿 机制下政府与银行的演化博亦、合作博亦等问题进 行探讨。例如, 杨昌兵和张目 (2017) ${ }^{[1]}$ 以江苏省 13 个市为例, 利用面板数据联立方程组模型实证检 验了政府设立科技型中小企业贷款风险补偿资金对 科技型中小企业获取银行贷款的影响。段义金和段 进东（2019） ${ }^{[2]}$ 采用 BC 2 模型测算苏南 11 个城市科 技贷款风险补偿资金池运营效率, 并结合 Malmquist 指数法对运营效率指数的变化进行分解, 并应用 Tobit 模型分析运营效率的影响因素。陈华丰和张目 （2017） [3]建立了政府与银行的演化博峦模型对货 款风险补偿资金的设立进行了探讨, 分析了模型的 8 种演化情况的稳定性。孙雅芳 (2016) [4]、Chen 和 Zhang（2016） ${ }^{[5]}$ 构建科技型中小企业贷款风险补偿 机制下政府、银行与科技型中小企业三方之间的合 作博亦模型, 并运用合作博亦的 Shapley 解, 对合作 收益分配情况进行分析, 期望得出提高科技型中小 企业贷款风险补偿资金使用效率的最佳比例, 使得 联盟合作机制能够长效运作, 并进行博亦仿真研究。 然而, 上述研究均未涉及科技型中小企业贷款风险 补偿机制中政府对银行的委托代理激励问题。事实 上，科技型中小企业贷款风险补偿机制中政府与银 行的委托代理关系本质上是一种多任务委托代理关 系, 其中, 银行有两项工作任务: 一是贷款发放; 二是呆账核销管理。在两项工作任务中, 银行在完 成贷款发放任务过程中的努力程度可以由政府直接 观测到, 而银行在完成呆账核销管理任务过程中的 努力程度是不可直接观测到的, 这就出现了对同一 代理人的不同工作监督难易程度的不同, 此时, “对
易于监督工作的过度激励就会诱使代理人将过多的 努力花在这些方面而忽视在其他方面的努力, 从而 导致激励本身的扭曲和低效” ${ }^{[6]}$ 。

Holmstrom 和 Milgnom（1991）[7]提出了多任务 委托代理模型, 证明了在代理人从事多项工作时, 从简单的委托-代理模型得出的结论是不适用的。该 模型认为, 当代理人从事性质不同的多种工作时会 给监督和激励带来更大的困难，代理人在不同任务 之间存在精力分配上的冲突, 因此, 对代理人的激 励应综合考虑不同任务的相互影响。有鉴于此, 本 文以 Holmstrom 和 Milgnom 的多任务委托代理模型 为基础, 构建银行在科技型中小企业贷款风险补偿 机制中的多任务委托代理模型, 并运用于对银行激 励问题的分析, 期望得出在此条件下的最优激励合 约安排，从而为建立健全科技型中小企业贷款风险 补偿机制中政府对银行的激励机制提供理论依据。

\section{2. 多任务委托代理模型的构建}

假设 1 科技型中小企业贷款风险补偿机制中银 行的工作任务至少包含以下两项：贷款发放和呆账 核销管理。

信贷是银行利用自身实力和信誉为客户提供资 金融通或代客户承担债务，并以客户支付利息、费 用和偿还本金或最终承担债务为条件的一种经营行 为。信贷业务基本操作流程包括受理、调查评价、 审批、发放及贷后管理五大阶段 ${ }^{[8]}$ 。其中, 贷款发 放包括落实贷前条件、签订合同、落实用款条件、 支用、信贷登记等五个步骤; 贷后管理包括对信贷 资产的检查、回收、展期、借新还旧、不良资产管 理等内容。而不良资产管理又包括日常管理、法律 手段追索、贷款重组、收取抵债资产、呆账核销等 内容。其中, 呆账核销是指银行经过内部审核确认 后, 动用呆账准备金将无法收回或者长期难以收回 的贷款或投资从账面上冲销，从而使账面反映的资 产和收入更加真实 ${ }^{[9]}$ 。《金融企业呆账核销管理办法 (2013 修订版)》第十六条规定: “金融企业要完善 呆账核销授权机制，明确股东大会、董事会和经营 管理层职责，按呆账核销政策要求，健全内部管理 制度，规范审核程序，及时核销呆账，并有效防范 虚假核销等各类风险。”显然，在科技型中小企业贷 款风险补偿机制中，委托人（政府）希望代理人 （银行）增加贷款发放数量和加强呆账核销管理。

设 $a=\left(a_{1}, a_{2}\right)$ 表示银行的努力向量, 其中, $a_{1}$ 是花在贷款发放任务上的努力, $a_{2}$ 是花在呆账核销 
管理任务上的努力; 用 $B\left(a_{1}, a_{2}\right)$ 表示努力的期望收 益（所有权属于委托人 $\left.{ }^{[10]}\right)$; 用 $C\left(a_{1}, a_{2}\right)$ 表示努力的 成本。假定 $B\left(a_{1}, a_{2}\right)$ 是严格递增的凹函数, $C\left(a_{1}, a_{2}\right)$ 是严格递增的凸函数 $\left[{ }^{[6]}\right.$ 。

当银行的努力选择为 $a=\left(a_{1}, a_{2}\right)$ 时, 决定了如下 可观测的信息向量 $x$ :

$$
x=\mu\left(a_{1}, a_{2}\right)+\varepsilon
$$

假定 $\mu: R_{+}^{2} \rightarrow R^{k}$ 是凹函数, 其中, $R$ 表示实数 域, $R^{k}$ 是 $k$ 维欧氏空间 $(k \geq 0)$, 在这里 $k=2 。 k$ 表示可观测信息的数量, 即 2 个努力变量决定了 2 个 可观测信息的数量。 $\varepsilon$ 是服从正态分布的随机向量, 其均值为 0 , 协方差矩阵为 $\Sigma$, 即 $\varepsilon_{1} \square N\left(0, \sigma_{1}^{2}\right)$, $\varepsilon_{2} \square N\left(0, \sigma_{2}^{2}\right)$ 。因此, $x$ 服从均值向量为 $\mu\left(a_{1}, a_{2}\right)$ 、 协方差矩阵为 $\Sigma$ 的正态分布。

假设 2 银行在不同工作任务上的努力产生不同 的业绩信息，且信息之间相互独立。

在假设 2 成立的条件下，信息向量 $x$ 可表示为

$$
x=\left[\begin{array}{l}
x_{1} \\
x_{2}
\end{array}\right], \quad x_{1}=\mu_{1}\left(a_{1}\right)+\varepsilon_{1}, \quad x_{2}=\mu_{2}\left(a_{2}\right)+\varepsilon_{2}
$$

上式表明了不同的努力变量产生不同的信息, 即 $x_{1}$ 反映了 $a_{1}$, 可以理解为观测到的贷款发放信息; $x_{2}$ 反映了 $a_{2}$, 可以理解为观测到的呆账核销管理信息。

假设 3 委托人 (政府) 是风险中性的, 代理人 (银行) 是风险规避的。银行具有统一的不变绝对 风险规避的效用函数, 即 $u=-\exp (-\rho \omega)$, 其中, $\rho$ 为绝对风险规避度, $\omega$ 为银行的实际货币收入。 努力成本 $C\left(a_{1}, a_{2}\right)$ 可为货币等价物。

设银行的报酬函数为线性形式, 即有

$$
s(x)=\alpha+\beta_{1} x_{1}+\beta_{2} x_{2}=\alpha+\beta^{T} x
$$

其中, $\alpha$ 为银行的基本报酬部分, 如合同规定的贷 款损失准备补偿 (或贷款利息补偿); $\beta^{T}=\left(\beta_{1}, \beta_{2}\right)$ 为业绩报酬系数向量。银行在双重任务下的确定性 等价收入 (certainty equivalence, CE) [6]为:

$$
\begin{aligned}
& C E=E(s(x))-\frac{1}{2} \rho \beta^{T} \Sigma \beta-C\left(a_{1}, a_{2}\right) \\
& =\alpha+\beta^{T} \mu\left(a_{1}, a_{2}\right)-\frac{1}{2} \rho \beta^{T} \Sigma \beta-C\left(a_{1}, a_{2}\right)
\end{aligned}
$$

其中, $E(s(x))$ 为期望报酬, $\frac{1}{2} \rho \beta^{T} \Sigma \beta$ 为收入的风险 贴水或风险成本。

由于政府是风险中性的, 其确定性等价收入就 是政府在多任务委托代理中的期望 “净收益”, 即

$$
B\left(a_{1}, a_{2}\right)-E\left(\alpha+\beta^{T} x\right)=B\left(a_{1}, a_{2}\right)-\alpha-\beta^{T} \mu\left(a_{1}, a_{2}\right)
$$

那么, 银行和政府的总的确定性等价收入（total certainty equivalence, TCE) 为:

$$
T C E=B\left(a_{1}, a_{2}\right)-\frac{1}{2} \rho \beta^{T} \Sigma \beta-C\left(a_{1}, a_{2}\right)
$$

若银行的保留效用为 $[u$, 则其参与约束条件
(IR) 为:

$$
C E \geq u
$$

基本报酬 $\alpha$ 对于银行而言, 没有任何激励作用, 其大小并不影响 $\beta^{T}$ 和 $\left(a_{1}, a_{2}\right)^{[10]}$; 在给定 $\beta^{T}$ 和 $\left(a_{1}, a_{2}\right)$ 的条件下, 基本报酬 $\alpha$ 由银行的保留效用 $\square u$ 决定 ${ }^{[6]}$ 。因此, 政府面临的决策就是如何选择适当 的 $\beta^{T}=\left(\beta_{1}, \beta_{2}\right)$ 来最大化总的确定性等价收入 ( TCE), 并且要满足银行的激励相容约束条件 (IC):

$$
\left(a_{1}, a_{2}\right) \in \arg \max \left(\beta^{T} \mu\left(a_{1}, a_{2}\right)-C\left(a_{1}, a_{2}\right)\right)
$$

为简单起见，假定 $\mu\left(a_{1}, a_{2}\right)=\left(a_{1}, a_{2}\right)^{T}$, 即观测 变量为 $x_{i}=a_{i}+\varepsilon_{i}, i=1,2$ 。如果所有的 $a_{i}$ 严格为正, 式（8）表示的激励相容约束条件就简化为其一阶条 件 ${ }^{[6]}$ :

$$
\beta_{i}=\frac{\partial C\left(a_{1}, a_{2}\right)}{\partial a_{i}}=C_{i}\left(a_{1}, a_{2}\right), \quad i=1,2
$$

式（9）隐含地决定了银行的努力函数 $a_{i}=a_{i}\left(\beta^{T}\right)$ 。 对式 (9) 求导, 并根据逆函数的定义, 有

$$
\frac{\partial \beta}{\partial a}=\left[C_{i j}\right] \text { 和 } \frac{\partial a}{\partial \beta}=\left[C_{i j}\right]^{-1}
$$

其中

$$
\frac{\partial \beta}{\partial a}=\left[\begin{array}{ll}
\frac{\partial \beta_{1}}{\partial a_{1}} & \frac{\partial \beta_{1}}{\partial a_{2}} \\
\frac{\partial \beta_{2}}{\partial a_{1}} & \frac{\partial \beta_{2}}{\partial a_{2}}
\end{array}\right], \quad\left[C_{i j}\right]=\left[\begin{array}{ll}
C_{11} & C_{12} \\
C_{21} & C_{22}
\end{array}\right]
$$

$\frac{\partial \beta}{\partial a}=\left[C_{i j}\right]$ 表示银行努力水平的单位变化所带来 的业绩报酬的变化, 相当于银行业绩报酬的变化对 其努力水平变化的敏感性; $\frac{\partial a}{\partial \beta}=\left[C_{i j}\right]^{-1}$ 则表示了政 府给予银行的业绩报酬的单位变化所引致的银行努 力水平的变化, 相当于银行努力水平的变化对业绩 报酬变化的敏感性。

令总的确定性等价收入（TCE）对 $\beta$ 的一阶导 数为 0 , 则有

$$
\begin{gathered}
\frac{\partial B}{\partial a}\left[\frac{\partial a}{\partial \beta}-\rho \sum \beta-\frac{\partial C}{\partial a}\left[\frac{\partial a}{\partial \beta}=0\right.\right. \\
\frac{\partial B}{\partial a}-\rho \frac{\partial \beta}{\partial a} \sum \beta-\frac{\partial C}{\partial a}=0 \\
B^{\prime}-\rho\left[C_{i j}\right] \Sigma \beta-\beta=0 \\
\left(I+\rho\left[C_{i j}\right] \Sigma\right) \beta=B^{\prime}
\end{gathered}
$$

上式两边同时左乘 $\left(I+\rho\left[C_{i j}\right] \Sigma\right)^{-1}$, 可得最大化总的 确定性等价收入 (TCE) 的一阶条件为:

$$
\beta=\left(I+\rho\left[C_{i j}\right] \Sigma\right)^{-1} B^{\prime}
$$

其中, $I$ 为单位阵, $B^{\prime}=\left(B_{1}, B_{2}\right)^{T}$ 为一阶偏导数向量, 即 $B_{i}=\frac{\partial B}{\partial a_{i}}$ 是第 $i$ 种工作任务上努力的边际收益; $C_{i j}$ 表示努力成本的二阶导数矩阵, 其经济含义是不 
同任务之间的关系 ${ }^{[11]} ; \Sigma$ 的经济含义是监督的难度， $\Sigma$ 越大, 难度越大 ${ }^{[11]}$ 。监督难度的产生可能是因为 业绩指标设置不够全面, 或某些业绩指标较难量化 (如呆账核销管理质量), 也有可能是因为委托人难 以获得代理人的真实信息。

\section{3. 多任务相互独立下的银行激励问题研究}

$\frac{\partial \beta}{\partial a}=\left[C_{i j}\right]$ 表示银行努力水平的单位变化所带来 的业绩报酬的变化, 也可以理解为在银行的单位努 力下, 政府对银行的激励成本变化率 ${ }^{[12]}$ 。由于呆账 核销管理所包含内容的复杂性, 以及贷款发放与呆 账核销管理之间相互影响的时滞性, 在某些特定时 期 (如政府与银行合作的初期), 两项工作任务可能 具有相对独立性, 其产出业绩信息也具有相对独立 性, 因此, 有如下假设:

假设 4 银行完成两项工作任务努力的激励成本 函数相互独立, 即 $C_{12}=C_{21}=0$, 且影响工作业绩信 息向量的随机向量 $\varepsilon$ 独立分布。

命题 1 如果银行具有相互独立的两项工作任务, 且两项工作任务努力的激励成本函数相互独立, 那 么在激励相容条件下, 两项工作任务的最优业绩报 酬 $\beta_{i}$ 也相互独立, 且 $\beta_{i}$ 是绝对风险规避度 $\rho$ 、边际 激励成本变化率 $C_{i i}$ 和可观测变量方差 $\sigma_{i}^{2}$ 的减函数。

证明: 由假设 4 可知, $C_{i j}=0(i \neq j)$, 且 $\Sigma$ 是 对角阵, 即有

$$
\frac{\partial \beta}{\partial a}=\left[\begin{array}{ll}
\frac{\partial \beta_{1}}{\partial a_{1}} & \frac{\partial \beta_{1}}{\partial a_{2}} \\
\frac{\partial \beta_{2}}{\partial a_{1}} & \frac{\partial \beta_{2}}{\partial a_{2}}
\end{array}\right]=\left[\begin{array}{cc}
\frac{\partial \beta_{1}}{\partial a_{1}} & 0 \\
0 & \frac{\partial \beta_{2}}{\partial a_{2}}
\end{array}\right]=\left[\begin{array}{cc}
C_{11} & 0 \\
0 & C_{22}
\end{array}\right]
$$

将式（13）代入式（12）, 则有

$$
\left[\begin{array}{l}
\beta_{1} \\
\beta_{2}
\end{array}\right]=\left[\left[\begin{array}{ll}
1 & 0 \\
0 & 1
\end{array}\right]+\rho\left[\begin{array}{cc}
C_{11} & 0 \\
0 & C_{22}
\end{array}\right]\left[\begin{array}{cc}
\sigma_{1}^{2} & 0 \\
0 & \sigma_{2}^{2}
\end{array}\right]\right]^{-1}\left[\begin{array}{c}
B_{1} \\
B_{2}
\end{array}\right]=\left[\begin{array}{c}
\frac{B_{1}}{\rho C_{11} \sigma_{1}^{2}+1} \\
\frac{B_{2}}{\rho C_{22} \sigma_{2}^{2}+1}
\end{array}\right]
$$

式（14）的通式为

$$
\beta_{i}=\frac{B_{i}}{\rho C_{i i} \sigma_{i}^{2}+1}, \quad i=1,2
$$

由式 (15) 可知, 如果银行具有相互独立的两 项工作任务, 且两项工作任务努力的激励成本函数 相互独立, 那么在激励相容条件下, 两项工作任务 的最优业绩报酬 $\beta_{i}$ 也相互独立, 即银行在贷款发放 任务上的最优努力独立于呆账核销管理任务上的最 优努力。同时, 最优业绩报酬 $\beta_{i}$ 是政府边际收益 $B_{i}$ 的增函数, 是银行绝对风险规避度 $\rho$ 、边际激励成 本变化率 $C_{i i}$ 和可观测变量方差 $\sigma_{i}^{2}$ 的减函数。命题 1 证毕。

$\beta_{i}$ 是政府边际收益 $B_{i}$ 的增函数, 意味着如果政
府在银行投入努力 $a_{i}$ 完成任务 $i$ 时获得的边际收益越 大, 则政府给予银行的业绩报酬也要增加, 反之亦 然。由于 $B\left(a_{1}, a_{2}\right)$ 是严格递增的凹函数, 所以政府 在科技型中小企业贷款业务规模的快速增长阶段应 给予银行相应递增的业绩报酬。

$\beta_{i}$ 是银行绝对风险规避度 $\rho$ 的减函数, 意味着 对于不同风险偏好的银行应该有不同的最优激励方 案, 但实践中会出现为了满足不同银行的个性化需 求而在激励政策上出现不统一的情形, 这是银行激 励问题上的一个难题。另外, 新近合作的银行由于 与政府的合作时间较短, 相互信任程度不高, 因此, 相对于长期合作的银行而言, 其绝对风险规避度 $\rho$ 相对较高, 那么, $\beta_{i}$ 是银行绝对风险规避度 $\rho$ 的减 函数所隐含的另一层政策含义是，对于刚刚建立合 作关系的银行应减少其业绩激励, 对于具有长期合 作关系的银行应增加其业绩激励。

$\beta_{i}$ 是边际激励成本变化率 $C_{i i}$ 的减函数所揭示 的政策含义在于, 如果银行某项工作任务努力的单 位激励成本高, 则应相应降低对其业绩的激励; 如 果银行某项工作任务努力的单位激励成本低, 则应 相应提高对其业绩的激励。从工作特性来看, 与呆 账核销管理工作相比，贷款发放工作的操作相对简 单, 且周期较短; 而呆账核销管理工作的操作相对 繁琐, 且周期较长, 因此, 银行在贷款发放任务上 努力的单位激励成本低于在呆账核销管理任务上努 力的单位激励成本，这样，政府将提高对贷款发放 任务业绩的激励, 降低对呆账核销管理任务业绩的 激励。

$\beta_{i}$ 是可观测变量方差 $\sigma_{i}^{2}$ 的减函数所揭示的政 策含义在于, 如果某项工作任务的可观测变量方差 高, 则应相应降低对其业绩的激励; 如果某项工作 任务的可观测变量方差低, 则应相应提高对其业绩 的激励。当某项工作任务的可观测变量方差较高时, 则意味着银行在该项工作任务上的努力与所创造的 业绩大小相关程度不高, 此时, 政府提高其业绩激 励可能达不到预期效果。只有当某项工作任务的可 观测变量方差较低时, 才能通过提高其业绩激励达 到激励其努力工作的效果。由于呆账核销管理质量 不易监督, 较难量化, 使得呆账核销管理任务的可 观测变量方差较高; 而贷款发放任务的完成情况及 其评价, 是由政府直接观测和度量的, 其可观测变 量方差近似于零。因此, 政府将降低对呆账核销管 理任务业绩的激励, 提高对贷款发放任务业绩的激 励。

\section{4. 多任务相互依存下的银行激励问题研究}

在假设 4 中作了银行完成两项工作任务努力的 激励成本函数相互独立的假设, 这符合某些特定时 期的观察事实。但我们也可以观察到, 从中长期来 看, 银行在贷款发放与呆账核销管理之间存在精力 分配上的冲突, 因此, 对银行的激励应综合考虑不 
同任务的相互影响。在此情况下，银行完成两项工 作任务努力的激励成本函数是相互依存的，即 $C_{12}=C_{21} \neq 0$, 因此, 有如下假设:

假设 5 银行完成两项工作任务努力的激励成本 函数相互依存, 即 $C_{12}=C_{21} \neq 0$, 且影响工作业绩信 息向量的随机向量 $\varepsilon$ 独立分布。

在两项工作任务中，银行在完成贷款发放任务 过程中的努力程度可以由政府直接观测到, 这是因 为政府通过贷款损失准备补偿（或贷款利息补偿） 申请报告就可以直接掌握银行在贷款发放方面付出 的努力; 而银行在完成呆账核销管理任务过程中的 努力程度是不可直接观测到的, 只有通过观测变量 $x_{2}=\mu_{2}\left(a_{2}\right)+\varepsilon_{2}$ 进行间接测度。为此, 有如下假设:

假设 6 在银行的两项工作任务中, 完成贷款发 放任务的努力选择是可以由政府直接观测到的, 即 $\sigma_{1}^{2}=0$; 而完成呆账核销管理任务的努力选择是政 府不可直接观测到的, 只有通过观测变量 $x_{2}=\mu_{2}\left(a_{2}\right)+\varepsilon_{2}$ 进行间接测度。

命题 2 如果银行完成两项工作任务努力的激励 成本函数相互依存, 那么在激励相容条件下:

（1）贷款发放任务的最优激励合约是 “门槛型 激励合约”, 即只有当政府在银行贷款发放任务努力 下的边际收益超过一定的 “门槛值” 时, 对其的激 励才是正向的, 否则将是负向的; 并且 “门槛值” 与银行两项工作任务努力的边际激励成本替代率 $C_{12}$ 、 呆账核销管理任务下的政府边际收益 $B_{2}$ 成正比, 与 呆账核销管理任务的边际激励成本变化率 $C_{22}$ 成反比。

(2) 呆账核销管理任务的最优激励合约与两项 工作任务努力的激励成本函数的相互依存性无关。

证明：由假设 6 可知, $\sigma_{1}^{2}=0$, 将其代入式 (12)，则有

$$
\begin{aligned}
& {\left[\begin{array}{l}
\beta_{1} \\
\beta_{2}
\end{array}\right]=\left[\left[\begin{array}{ll}
1 & 0 \\
0 & 1
\end{array}\right]+\rho\left[\begin{array}{ll}
C_{11} & C_{12} \\
C_{21} & C_{22}
\end{array}\right]\left[\begin{array}{cc}
0 & 0 \\
0 & \sigma_{2}^{2}
\end{array}\right]\right]^{-1}\left[\begin{array}{l}
B_{1} \\
B_{2}
\end{array}\right]} \\
& \beta_{1}=B_{1}-\frac{\rho C_{12} \sigma_{2}^{2} B_{2}}{1+\rho C_{22} \sigma_{2}^{2}} ; \quad \beta_{2}=\frac{B_{2}}{1+\rho C_{22} \sigma_{2}^{2}}
\end{aligned}
$$

由 $\beta_{1}=B_{1}-\frac{\rho C_{12} \sigma_{2}^{2} B_{2}}{1+\rho C_{22} \sigma_{2}^{2}}$ 可知, 贷款发放任务的最 优激励合约是 “门槛型激励合约”, 即只有当政府在 银行贷款发放任务努力下的边际收益满足下式时, 对这一任务的激励才是正向的, 否则将是负向的。

$$
B_{1}>\frac{\rho C_{12} \sigma_{2}^{2} B_{2}}{1+\rho C_{22} \sigma_{2}^{2}}
$$

同时, 通过分析上述门槛条件可知, 门槛条件 与银行两项工作任务努力的边际激励成本替代率 $C_{12}$ 、 呆账核销管理任务努力下的政府边际收益 $B_{2}$ 成正比, 与呆账核销管理任务努力的边际激励成本变化率 $C_{22}$ 成反比。命题 2 中的（1）得证。

由 $\beta_{2}=\frac{B_{2}}{1+\rho C_{22} \sigma_{2}^{2}}$ 可知, $C_{12}$ 并未出现在等式中,
因此，呆账核销管理任务的最优激励合约与两项工 作任务努力的激励成本函数的相互依存性无关。命 题 2 中的 (2) 得证。

命题 2 的经济意义在于, 当银行两项工作任务 努力的激励成本函数相互依存 $\left(C_{12}=C_{21} \neq 0\right)$, 且 贷款发放任务的努力选择可以直接观测 $\left(\sigma_{1}^{2}=0\right)$ 时, 对呆账核销管理任务的最优激励报酬 $\beta_{2}$ 与两项 工作任务努力的激励成本函数的相互依存性无关, 也即银行两项工作任务努力的边际激励成本替代率 $C_{12}$ (激励成本函数的交叉偏导 ${ }^{[13]}$ ) 并不影响呆账核 销管理任务的最优激励报酬 $\beta_{2}$ 的确定, 其最优化激 励条件与两项工作任务努力的激励成本函数相互独 立时的情形完全相同。

但是，对贷款发放任务的最优化激励条件则有 较大变化。从 $\beta_{1}$ 的取值上可以看出, 由于贷款发放 任务的努力选择可以直接观测, 其最优激励合约构 成一个 “门槛型激励合约”。而门槛条件与银行两项 工作任务努力的边际激励成本替代率 $C_{12}$ 、呆账核销 管理任务努力下的政府边际收益 $B_{2}$ 成正比，与呆账 核销管理任务努力的边际激励成本变化率 $C_{22}$ 成反比。 上述结论与观察事实相符。呆账核销管理质量较高 的银行, 其声誉良好, 呆账核销管理任务努力下政 府的边际收益较高, 政府将扩大与银行的合作, 银 行将承担更多的贷款发放任务，政府对贷款发放任 务的要求随之提高; 而呆账核销管理质量较差的银 行, 其声誉也较差, 政府将减少或中止与银行的合 作，政府对贷款发放任务的要求相应下降。结合式

（10）同样可以理解, 银行两项工作任务努力的边 际激励成本替代率 $C_{12}$ 越高, 意味着银行在呆账核销 管理任务努力下的贷款发放业绩报酬越高, 因此, 门槛条件也会相应提高。而门监条件与呆账核销管 理任务努力的边际激励成本变化率 $C_{22}$ 成反比, 则意 味着银行的单位呆账核销管理努力所要求的呆账核 销管理业绩剩余分享比例越高, 则政府在贷款发放 任务上的激励门槛会降低, 从而提高对银行在贷款 发放任务上的激励。

最优化激励条件 $\beta_{1}=B_{1}-\frac{\rho C_{12} \sigma_{2}^{2} B_{2}}{1+\rho C_{22} \sigma_{2}^{2}}$ 所揭示的经 济含义还包括: 在激励门槛条件未达到时, 对银行 在贷款发放任务上的激励将是负向的，将对银行的 贷款发放积极性产生不利影响; 但是，一旦超过门 槛值，则银行在贷款发放任务上所得到的激励将完 全由其在贷款发放方面所创造的增量业绩来决定, 而不是与呆账核销管理报酬激励一样是一种剩余索 取比例的分享 ${ }^{[13]}$, 这无疑会诱导银行把更多的精力 和时间放在贷款发放任务上，从而不利于呆账核销 管理质量的提高。

\section{5. 结论与政策含义}

本文运用多任务委托代理模型对科技型中小企 
业贷款风险补偿机制中政府对银行的激励问题进行 了研究, 得出如下结论：1）如果银行具有相互独立 的两项工作任务, 且两项工作任务努力的激励成本 函数相互独立, 那么在激励相容条件下, 两项工作 任务的最优业绩报酬也相互独立, 且最优业绩报酬 是绝对风险规避度、边际激励成本变化率和可观测 变量方差的减函数。2) 如果银行完成两项工作任务 努力的激励成本函数相互依存, 那么在激励相容条 件下，贷款发放任务的最优激励合约是 “门槛型激 励合约”, 即只有当政府在银行贷款发放任务努力下 的边际收益超过一定的 “门槛值” 时, 对其的激励 才是正向的, 否则将是负向的; 并且 “门槛值” 与 银行两项工作任务努力的边际激励成本替代率、呆 账核销管理任务下的政府边际收益成正比，与呆账 核销管理任务的边际激励成本变化率成反比。而呆 账核销管理任务的最优激励合约与两项工作任务努 力的激励成本函数的相互依存性无关。

贷款业务的特性决定了在科技型中小企业贷款 风险补偿机制中银行至少有两项工作任务: 一是贷 款发放; 二是呆账核销管理。由于两项工作任务完 成情况的可观测变量、努力选择可观测程度不一致, 求解出的银行最优化激励条件, 在激励成本函数相 互独立和相互依存两种情形下均明确显示: 理性的 参与人一一政府和银行, 都会被现实所诱导, 用更 多的精力和时间去完成贷款发放任务, 而花在呆账 核销管理任务上的努力则相对较少。这样, 势必会 导致政府对银行的财政激励的扭曲和低效。因此, 政府有必要建立银行呆账核销管理质量评估体系, 对银行在完成呆账核销管理任务过程中的努力程度 进行直接观测。在此基础上, 政府可以建立贷款发 放业绩和呆账核销管理业绩并重的银行激励机制。 具体思路包括：1）根据贷款发放业绩, 建立科技型 中小企业贷款风险补偿奖励累进制度。例如, 以上 一年度银行对科技型中小企业贷款季均余额为基数, 本年度科技型中小企业贷款季均余额超基数部分, 按累进比例给予风险补偿奖励; 风险补偿奖励主要 用于充实银行的呆账准备金，银行可根据实际情况， 提取不高于一定比例的风险补偿奖励对科技型中小 企业贷款相关从业人员予以奖励。2）根据呆账核销 管理业绩, 设置差异化的科技型中小企业贷款呆账 补偿标准。例如, 各级财政部门和银行业监督管理 部门在银行呆账核销的事后监督和管理中, 根据银 行呆账核销管理制度的完备性及其实施情况, 以及 呆账认定、核销中违规违法行为的发生情况等, 对 同级银行呆账核销管理质量进行全面评估, 根据评 估结果, 设置差异化的科技型中小企业贷款呆账补 偿标准, 对呆账核销管理质量较高的银行给予较高 的补偿比例, 对呆账核销管理质量较差的银行给予 较低的补偿比例。

\section{致谢}

This research was funded by the Regional Project of National Natural Science Foundation of China, grant number 71263011 and 71861003.

\section{参考文献}

[1] 杨昌兵,张目.科技型中小企业贷款风险补偿资金 的杜杆效应研究 [J].科技创业月刊,2017,30(13):3537.

[2] 段义金䎴进东.江苏科技贷款风险补偿资金池运 营效率测评一一基于 BC 2 模型和 Malmquist 指 数法[J].科技管理研究,2019,39(17):63-70.

[3] 陈华丰,张目.贷款风险补偿机制实施中政府与银 行间的演化博弯研究 [J]. 科技创业月 刊,2017,(10):97-100.

[4] 孙雅芳.科技型中小企业贷款风险补偿机制的博弯 分析[D].贵阳:贵州财经大学,2016.

[5] Chen H F, Zhang M. Simulation Research on Cooperation Game between Bank, Government and Enterprise under Risk Compensation Mechanism[C]. 2016 5th EEM International Conference on Education Science and Social Sciences. Sydney, Australia: IERI Advances in Education Research, 2016, 93: 217-221.

[6] 张维迎.博弯论与信息经济学 $[\mathrm{M}]$.上海:上海人民 出版社,2004.

[7] Holmstrom B, Milgrom P. Multi-task principal-agent analyses: Incentive contracts, asset ownership and job design[J]. Journal of Law, Economics and Organization, 1991,7(Special Issue): 24-52.

[8] 李斌,张海鹏. 商业银行信贷业务理论与实务 $[\mathrm{M}]$. 沈阳:白山出版社,2006.

[9] 姜占华.银行不良贷款的处置方式及法律简析 [J]. 网络财富,2009,(12):167-169.

[10] 吴庆田.企业年金基金投资管理人的激励机 制优化一一基于多任务委托代理模型的研究 [J]. 财经理论与实践,2010,31(2):40-44.

[11] 聂辉华.取消农业税对乡镇政府行为的影响: 一个多任务委托代理模型 [J]. 世界经 济,2006,29(8):71-78.

[12] 袁江天,张维.多任务委托代理模型下国企经 理激励问题研究 [J].管理科学学报,2006,9(3):45-53.

[13] 陈伟,但斌.考虑零售商多任务参与下的供应 链激励合约研究 [J]. 商业经济与管理,2010,(12):1017 . 